\title{
Truncation and composition of schedules: A good strategy for solving bicriteria scheduling problems
}

\author{
E. O. Oyetunji \\ Department of Computer Science Faculty of Computational and Developmental Mathematics \\ University for Development Studies, Ghana, eoyetunji@yahoo.com
}

\begin{abstract}
This paper consider the bicriteria scheduling problem of simultaneously minimizing the total completion time (Ctot) and number of tardy jobs (NT) with release dates on a single machine. A heuristic (called HR7) was proposed for solving this problem and was compared with a heuristic (called HR6) selected from the literature and a branch and bound (BB) method. The proposed heuristic makes use of the idea of truncation and composition of schedules. The three solution methods were tested on 1100 randomly generated problems ranging from 3 to 500 jobs. Performance evaluation, based on both effectiveness and efficiency of the solution methods, were carried out. Experimental results are provided.
\end{abstract}

Keywords: Scheduling; Bicriteria; Single machine, Release dates, Truncation and Composition of schedules

\section{INTRODUCTION}

The bicriteria scheduling problems, unlike the singlecriterion scheduling problems, involves optimization of two scheduling criteria or performance measures (Ehrgott and Grandibleux, 2000). The problems may be approached using different techniques (Hoogeveen, 2005). The two criteria may be optimized simultaneously if they both carry equal weights (that is the relative importance of the two criteria is the same). On the other hand, if one criterion is more important than the other, then the criterion that is less important is minimized subject to the constraint that the more important criterion is less or equal to a predetermined constant (for a minimization problem). The third approach deals with situation in which we are unable to categorically state that the two criteria are of same importance or that one criterion is more important than the other criterion. In this technique, a set of compromise solutions on both criteria are sought. This is called the pareto-optimal set.

In this paper, the simultaneous minimization approach is adopted. This is because it provides a true bicriteria approach to solving the bicriteria scheduling problems. There are three different approaches in simultaneous minimization (Evans, 1984; Fry, el al., 1989). These are: a priori optimization, interactive optimization, and a posteriori optimization. In the case of a priori optimization, both criteria are aggregated into a single function called the composite objective function (COF), then optimum solution (solution for which the COF yields an optimum value) is determined for the problem. This COF can either be linear or general (quadratic etc) function.

The interactive optimization requires the active involvement of a decision maker during the solution process. For example, given one or more already obtained solutions, the decision maker must indicate which one is preferable, and if not satisfied yet, in which direction the search should continue. In the case of posteriori optimization, we select from the set of solutions a subset that contains an optimum solution for each reasonable composite objective function that we can think of, then we present this set to the decision maker and let him/her choose the preferred solution.

In this paper, the priori approach is adopted. This is because most decision-maker does not want to be bothered with the rigorous analytical processes involved in both the interactive and posteriori approaches. To the best of our knowledge only Oyetunji (2006) and Oyetunji and Oluleye (2008a) have proposed heuristics for the bicriteria scheduling problem of simultaneously minimizing the total completion time and number of tardy jobs with release dates on a single machine. In 2006, Oyetunji proposed two heuristics (HR1 and AL1) for the single criterion scheduling problem of minimizing the total completion time with release dates on a single machine. His experimental results showed that AL1 outperformed others (HR1 and BESTA (selected from 
the literature). Also, Oyetunji (2006) proposed two heuristics (HR2 and HR3) for the single criterion scheduling problem of minimizing the number of tardy jobs with release dates on a single machine. Again, his experimental results showed that the DAU heuristic (selected from Dauzere-Perez (1995) outperformed the two proposed heuristics.

Oyetunji (2006) used the idea of Stein and Wein (1997) to propose three heuristics (HR4, HR5 and HR6) for the bicriteria scheduling problem of simultaneously minimizing the total completion time and number of tardy jobs with release dates on a single machine. Stein and Wein (1997) worked on "On the existence of schedules that are near-optimal for both makespan and total weighted completion time". They showed that given an optimal makespan schedule and an optimal total weighted completion time schedule, a valid schedule can be constructed from both schedules through a process called truncation and composition of schedules. The performance evaluation carried out on HR4, HR5 and HR6 show that the HR6 outperformed others. It was noted that the HR6 heuristic was made up of the AL1 heuristic (earlier adjudged as the best heuristic for the single criterion scheduling problem of minimizing the total completion time with release dates on a single machine) and DAU heuristic (also adjudged as the best heuristic for the single criterion scheduling problem of minimizing the number of tardy jobs with release dates on a single machine). This is in perfect agreement with the Stein and Wein's truncation and composition rule. This work was published in Oyetunji and Oluleye (2008a).

In an attempt to seek better solution method for the single criterion scheduling problem of minimizing the total completion time with release dates on a single machine, Oyetunji and Oluleye (2007) proposed a new heuristic (called AEO) which outperformed the AL1 heuristic. In 2008, Oyetunji and Oluleye (2008b) proposed a new heuristic (called EOO) for the single criterion scheduling problem of minimizing the number of tardy jobs with release dates on a single machine. Their experimental results showed that the EOO heuristic outperformed the DAU heuristic for all the considered problem sizes. The observation on the performance of HR6 (since it uses the best heuristics as at then for Ctot and NT) over HR4 and HR5 encouraged us to propose the HR7 heuristic (which uses the AEO and EOO heuristics) for the bicriteria scheduling problem of simultaneously minimizing the total completion time and number of tardy jobs with release dates on a single machine.

\section{MATERIALS AND METHODS:}

The materials and methods adopted in this work are described below.

THE PROBLEM: Given the following for a single machine problem:

i.a set of $n$ jobs; $J_{1}, J_{2}, \ldots J_{n}$

ii.processing time of each job; $P_{i}$

iii.release or ready time of each job; $r_{i}$

iv.due dates of each job; $d_{i}$

The time at which the processing of each jobs completes is $\mathrm{C}_{i}$. A job $\mathrm{J}_{i}$ is said to be tardy if it is completed after its due date (i.e. $C_{i}>d_{i}$ ).

We define

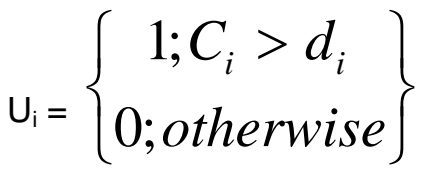

The aim is to simultaneously minimize the total completion time and number of tardy jobs criteria on a single machine with release time. We assume that pre-emption is not allowed and that the problem is static and deterministic i.e. number of jobs, their processing times, and ready times are all known and fixed. This assumption seems reasonable as many real life scheduling problems can be so modeled. For an example, in a manufacturing setting, no manager would want to commence operations (running expensive machines) when uncertain about the number of jobs, their processing requirements, due dates and their ready times.

The total completion time criterion takes care of the manufacturer's concern while the customer's concern is taken care of by the number of tardy jobs criterion. Hence, the bicriteria problem being considered addresses both the manufacturer's and customer's concerns simultaneously.

Using the notations of Graham et al. (1979), the problem is represented as

1| $\mathrm{r}_{\mathrm{i}} \mid\left(\sum_{i=1}^{n} C_{i}, \sum_{i=1}^{n} U_{i}\right)$.

Proposed Solution Methods: The problem of simultaneously minimizing the total completion time and number of tardy jobs criteria on a single machine with release time is NP-Hard (Nagar et al., 1995). Therefore, heuristics approaches are desired to obtain fairly good schedules. One heuristic (called HR7) is proposed for the bicriteria scheduling problem. 
HR7 Heuristic: In line with Stein and Wein (1997) rule of truncation and composition of schedules, the HR7 heuristic is a combination of the AEO heuristic (selected from Oyetunji and Oluleye (2007) and EOO heuristic (selected from Oyetunji and Oluleye (2008b) methods. The AEO method was proposed for the single criterion scheduling problem of minimizing the total completion time of jobs on a single machine with release time while the EOO method was proposed for the single criterion scheduling problem of minimizing the number of tardy jobs on a single machine with release time. The HR7 heuristic steps are outlined below:

\section{HR7 Heuristic Steps}

Step 1 Obtain a schedule (M1) using AEO.

Step 2 Obtain a schedule (M2) using EOO.

Step 3 Remove all late jobs from M2 and call the remaining schedule $\mathrm{M}^{1}$

Step 4 Obtain schedule $M 1^{1}$ by removing $M 2^{1}$ from M1

Step 5 Form a schedule HR7 by appending $M 1^{1}$ to the end of $M 2^{1}$. Note that $M 1^{1}$ should be appended in the ascending order of the processing times of the jobs in $\mathrm{M}^{1}$ schedule.

Selected Solution Methods: Based on its performance, a heuristic (called HR6) was selected from Oyetunji and Oluleye (2008a) for evaluation. Also selected for evaluation purposes is a branch and bound (BB) method implemented by Oyetunji and Oluleye (2008a). The HR6 heuristic systematically combines the AL1 and DAU heuristics. For details of the HR6 heuristic and the BB method, see pg. 149 of Oyetunji and Oluleye (2008a).

Data Analysis: In order to assess the performance of the proposed heuristic in relation to the selected solution methods, the same set of problems solved by Oyetunji and Oluleye (2008a) were solved. These consist of 50 problems each for 22 different problem sizes ranging from 3 to 500 jobs. In all, a total of 1100 randomly generated problems were solved. The processing time of the jobs were randomly generated with values ranging between 1 and 100 inclusive. The ready time of the jobs were also randomly generated with values ranging between 0 and $\sum_{i=1}^{n} P_{i}$ inclusive.

The due dates of the jobs were also randomly generated with values ranging between $\left(r_{i}+p_{i}\right)$ and $\left(r_{i}\right.$ $+2^{*} \mathrm{p}_{\mathrm{i}}$ ) inclusive.
A program was written in Microsoft visual basic 6.0 to apply the solution methods (HR7, HR6 and BB) to the problems generated. The program computes the value of the normalized linear composite objective function obtained by each solution method for each problem. The data was exported to Statistical Analysis System (SAS version 9.1) for detailed analysis. SAS is a very versatile statistical package and was employed to enable credible conclusions to be drawn from the results. The hardware used for the experiment is a $1.73 \mathrm{GHz}$ T2080 Intel CPU with 1024 $M B$ of main memory.

The general linear model (GLM) procedure in SAS was used to compute the mean value of the normalized linear composite objective function for each problem size (50 problem instances were solved under each problem size) and by solution methods. The test of means was also carried out using the GLM procedure so as to determine whether or not the differences observed in the mean value of the normalized linear composite objective function obtained by various solution methods are statistically significant. The methodology of Oyetunji (2009) was used to obtain the normalized linear composite objective function. Therefore, the normalized linear composite objective function shown in the results is dimensionless.

\section{RESULTS AND DISCUSSION:}

The mean values of the normalized linear composite objective function obtained from the various solution methods and for various problem sizes considered are shown in Table 1. Based on the minimum mean value of the normalized linear composite objective function, a ranking order of BB, HR7 and HR6 was obtained (Table 1). This means that with respect to effectiveness (closeness of the mean values of the normalized linear composite objective function to the optimal) the HR7 heuristic outperformed the HR6 heuristic for all the problem sizes considered.

In order to determine whether the differences observed in the mean values of the normalized linear composite objective function obtained from the various solution methods are significant or not, the results presented in Table 1 was subjected to statistical test. The results obtained are summarized in Tables 2-4. The differences in the mean value of the normalized linear composite objective function obtained from BB and HR7 solution methods are not significant (indicating competitive performances between BB and HR7) at 5\% level when the number of jobs is less than 30 (Table 2). However, under the 
same job configuration, the mean value of the normalized linear composite objective function obtained from the HR6 heuristic is significantly different (indicating poor performance compare with $B B$ and HR7) from that of BB and HR7 solution methods (Table 2).

When the number of jobs ranges between 30 and 200 inclusive, the differences in the mean value of the normalized linear composite objective function obtained from BB is significantly different (indicating better performance compare with HR7 and HR6) from that of HR6 and HR7 solution methods (Table 3). Also, under the same problem loading, the difference in the mean value of the normalized linear composite objective function obtained from HR7 is significantly different (indicating better performance compare with HR6) from that of HR6 heuristic (Table 3). Table 4 show that for problem sizes ranging from 300 to 500 jobs, the difference in the mean value of the normalized linear composite objective function obtained from HR7 is significantly different (indicating better performance compare with HR6) from that of HR6 heuristic. The BB procedure could not be applied to problems involving more than 200 jobs as it could not obtain solution within the maximum allowable time of 60 minutes due to its obvious implicit enumeration characteristic.

When the ratio of the values of the normalized linear composite objective function obtained from the HR6

\section{Table 1 Mean of the normalized composite objective functic}

\section{Problem}

Size

$3 \times 1$

$4 \times 1$

$5 \times 1$

$6 \times 1$

$7 \times 1$

$8 \times 1$

$9 \times 1$

10x1

$12 \times 1$

$15 \times 1$

$20 \times 1$

$25 \times 1$

$30 \times 1$

$40 \times 1$

$50 \times 1$

100x1

$120 \times 1$

$140 \times 1$

$200 \times 1$

$300 \times 1$

$400 \times 1$

$500 \times 1$

Sample size $=50$

\section{Mean of the normalized composite objective function}

\section{BB}

0.2868

0.3862

0.3616

0.3905

0.3971

0.4003

0.4215

0.4225

0.4180

0.4555

0.4755

0.4690

0.4612

0.4744

0.4857

0.4851

0.4853

0.4830

0.4804

-

\section{HR6}

0.4105

0.4518

0.4958

0.4719

0.4915

0.5291

0.5132

0.5069

0.4941

0.5197

0.5072

0.5037

0.5011

0.5109

0.5132

0.4990

0.5067

0.4967

0.4957

0.4955

0.4938

0.4930 and HR7 to that of the BB (HR6/BB and HR7/BB) were computed for the various problem sizes, the results obtained are shown in Fig. 1. The closeness of HR7's performance to that of BB when the number of jobs is less than 30 is obvious (within the 10\% range). The performance of HR6 is within $45 \%$ of that of the BB method (Fig. 1). Fig. 2 shows the approximation ratio of HR6 over HR7. The impressive performance of HR7 over HR6 especially when the number of jobs is less than 100 is obvious (Fig. 2).

The mean values of the execution time (seconds) taken to obtain results by the BB, HR6 and HR7 for various problem sizes considered are shown in Fig. 3. As expected, the BB method took longer time (slower) than the HR6 and HR7 heuristics (Fig. 3). Since the execution time of HR6 and HR7 were superimposed, the execution time (seconds) taken to obtain results by the HR6 and HR7 for various problem sizes considered were drawn separately and are shown in Fig. 4. The HR7 is slower than the HR6 when the number of jobs exceeds 40 . When the number of jobs is less than 40 , the plot of execution time of HR7 and HR6 still look superimposed. Therefore, in order to see clearly what is happening when the number of jobs is less or equal to 50 , a plot of execution time of HR6 and HR7 was repeated and is as shown in Fig. 5. It is now obvious that the HR7 is faster than the HR6 when the number of jobs is less than 30 (Fig. 5).

on solution methods and problem sizes 
Am. J. Sci. Ind. Res., 2010, 1(3): 427-434

Table 2 Test of means (probabily values) of normalized composite objective function for $3 \leq n<30$ problems

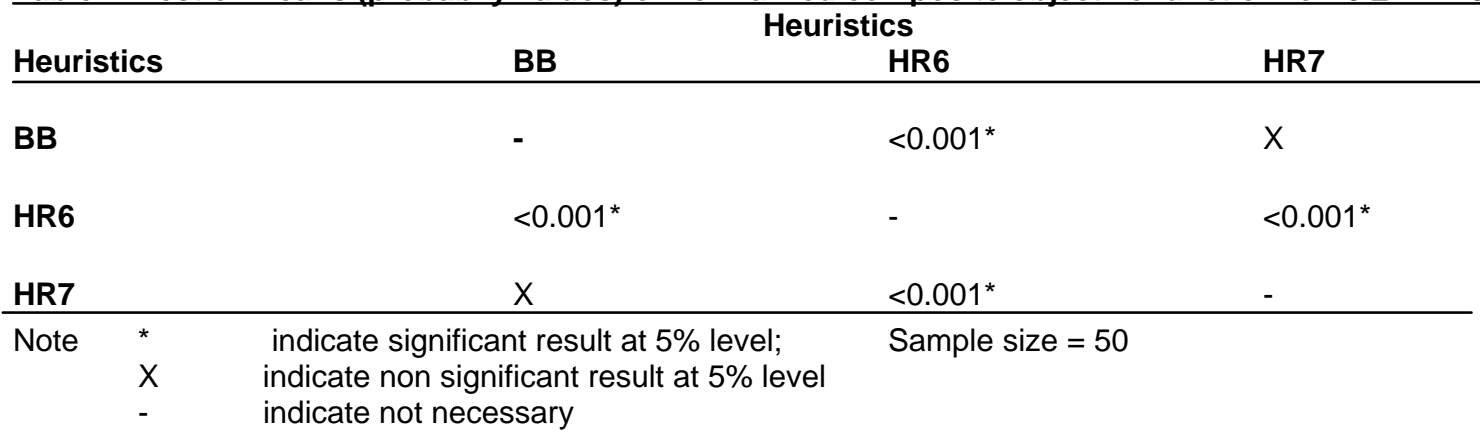

Table 3 Test of means (probabily values) of normalized composite objective function for $30 \leq n \leq 200$ problems Heuristics

\begin{tabular}{llll} 
Heuristics & BB & HR6 & HR7 \\
\hline BB & - & $<0.001^{*}$ & $<0.001^{*}$ \\
HR6 & $<0.001^{*}$ & - & $<0.001^{*}$ \\
HR7 & $<0.001^{*}$ & $<0.001^{*}$ & - \\
\hline Note $*$ & $\begin{array}{c}\text { indicate significant result at 5\% level; } \\
\text { indicate not necessary }\end{array}$ & Sample size $=50$ &
\end{tabular}

Table 4 Test of means (probabily values) of normalized composite objective function for $300 \leq n \leq 500$ problems Heuristics

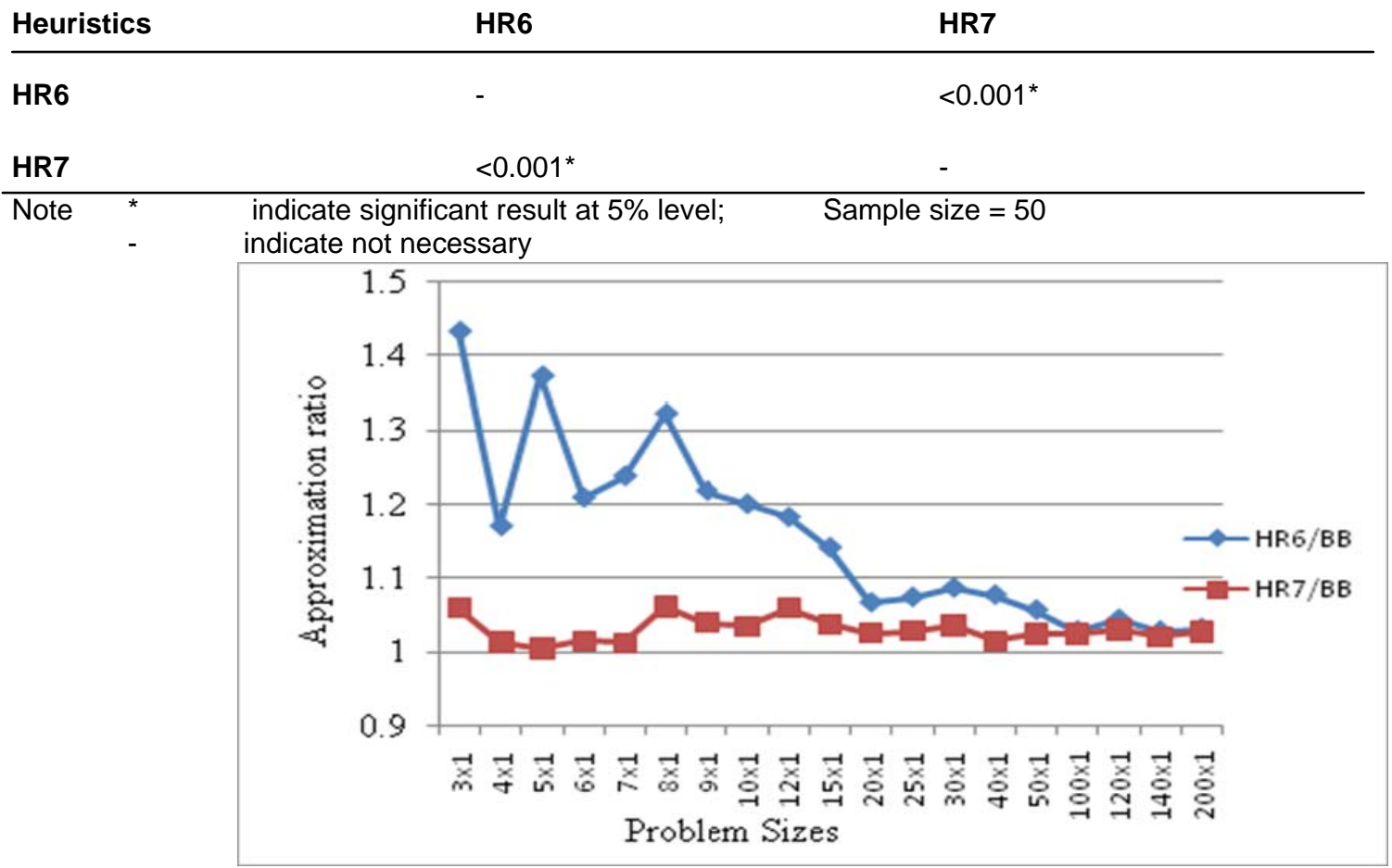

Fig. 1 Comparison of HR6 and HR7 with BB using approximation ratio 
Am. J. Sci. Ind. Res., 2010, 1(3): 427-434

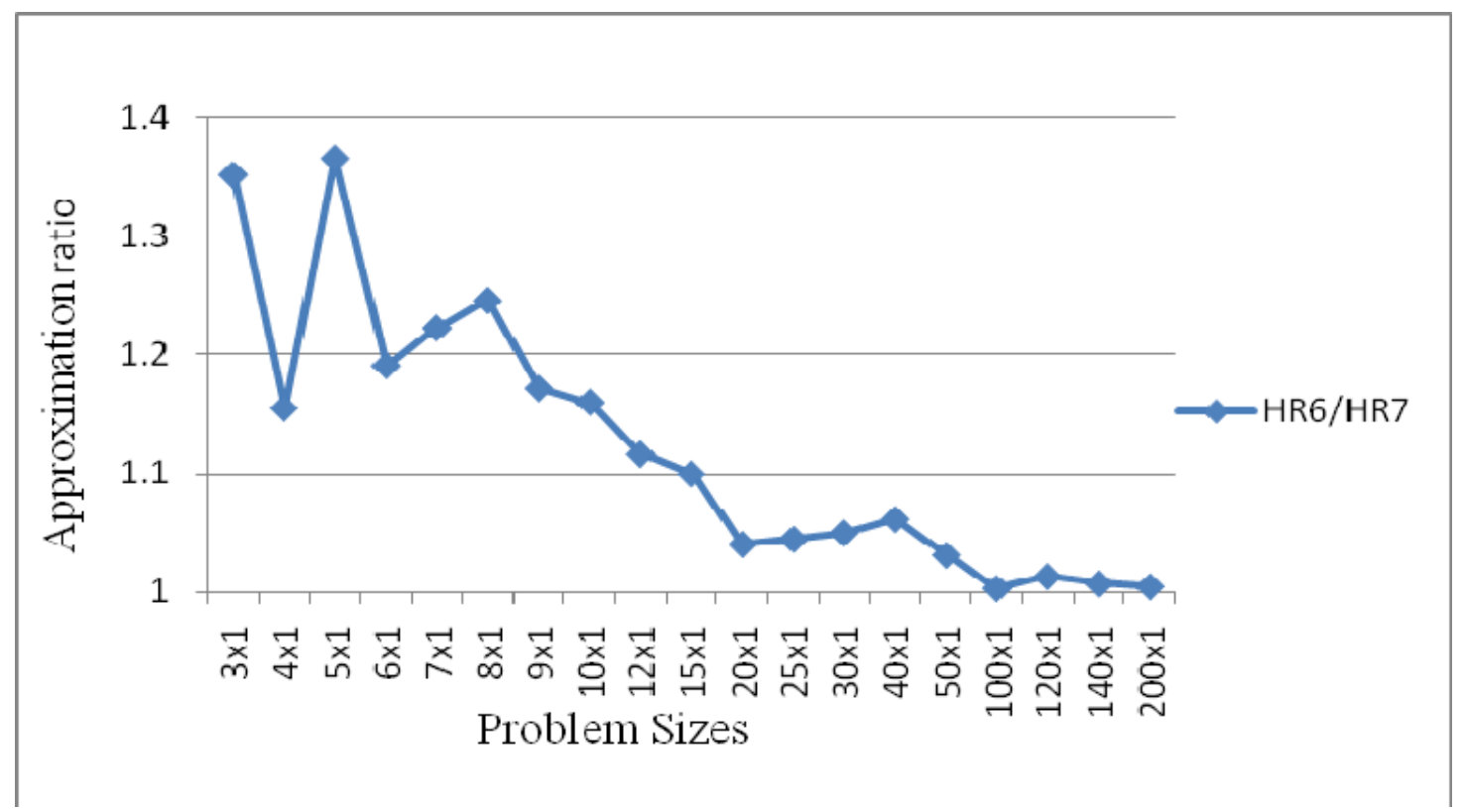

Fig. 2 Comparison of HR7 with HR6 using approximation ratio

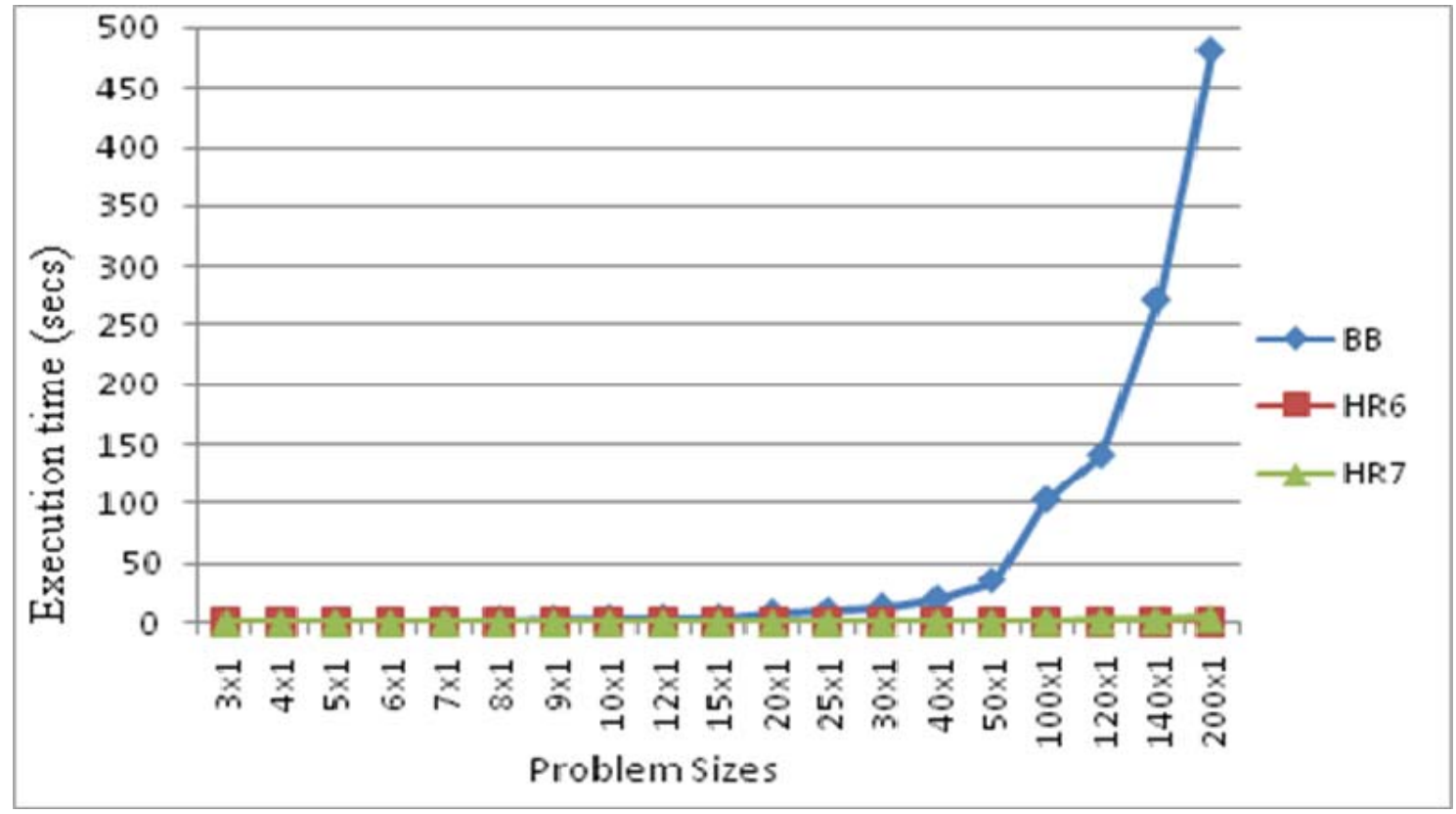

Fig. 3 Execution time of solution methods by problem sizes 
Am. J. Sci. Ind. Res., 2010, 1(3): 427-434

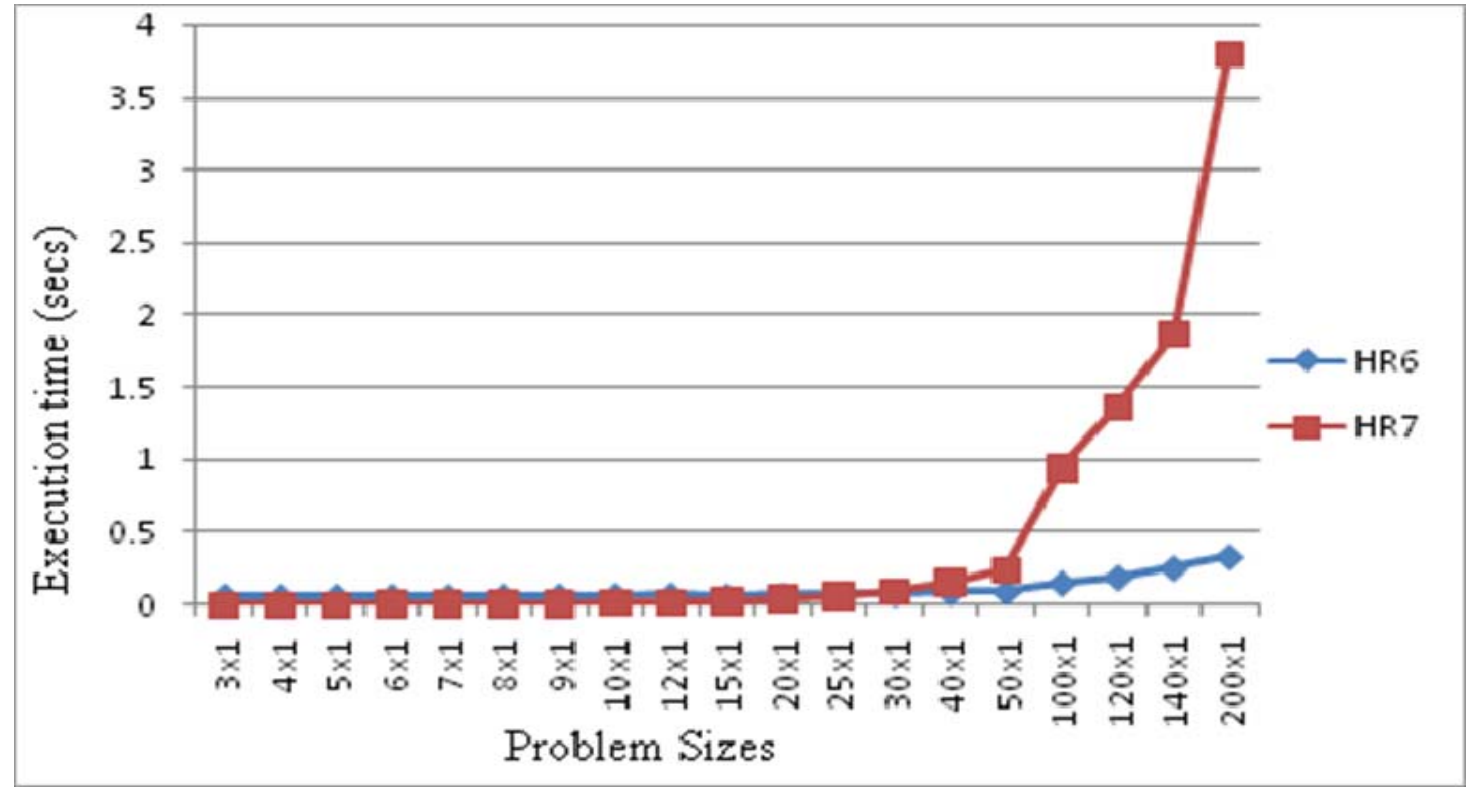

Fig. 4 Comparison of execution time of HR6 and HR7 for 3 to 200 problem sizes

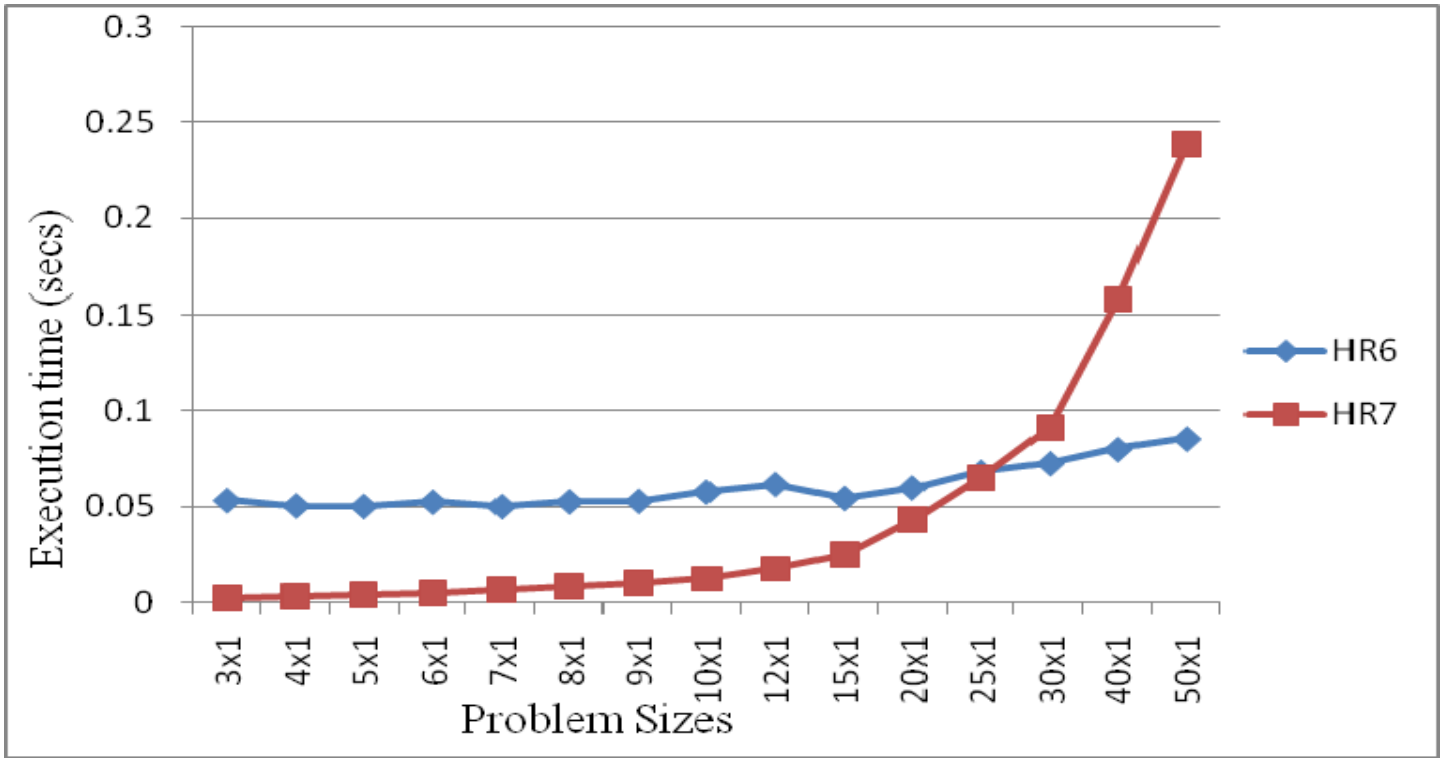

Fig. 5 Comparison of execution time of HR6 and HR7 for 3 to 50 problem sizes

\section{CONCLUSIONS:}

The bicriteria scheduling problem of simultaneously minimizing the total completion time and number of tardy jobs with release dates on a single machine has been explored. In the past, the idea of Stein and Wein's (1997) truncation and composition rule led to good heuristic solution. Stein and Wein's (1997) rule states that "given an optimal makespan schedule and an optimal total weighted completion time schedule, a valid schedule can be constructed from both schedules through a process called truncation and composition of schedules". Having made landmark progress in the single criterion problems involving total completion time and number of tardy jobs, Stein and Wein's (1997) idea has been used again to 
propose the HR7 heuristic for the bicriteria problem considered in this paper. In view of the impressive performance of the HR7 heuristic over the HR6 heuristic in terms of both effectiveness and efficiency, the HR7 heuristic is recommended for the bicriteria scheduling problem of simultaneously minimizing the total completion time and number of tardy jobs with release dates on a single machine. The consequences of the results obtained are as follows:

The use of Stein and Wein's idea of truncation and composition of schedules in a systematic way is recommended

Researchers are encouraged to continue the search for better solution methods for the single-criterion scheduling problems, as they could use Stein and Wein's idea to obtain better solution methods to solve many Bicriteria scheduling problems.

\section{REFERENCES}

Dauzere-Perez, S. (1995). Minimizing late jobs in the general one machine scheduling problem, European Journal of Operational Research, 81:134-142.

Ehrgott, M. and Grandibleux, X. (2000). An Annotated Bibliography of Multiobjective Combinatorial Optimization, Report in Wirtschaftsmathematik, No. 62(2000), Fachbereich Mathematik - Universitat Kaiserslautern.

Evans, G.W. (1984). An overview of techniques for solving multiobjective mathematical programs, Management Science, 30:1268-1282.

Fry, T.D., Armstrong, R.D. and Lewis, H. (1989). A framework for single machine multiple objective sequencing research, Omega 17:595-607.
Graham, R.L., Lawler, E.L., Lenstra, J.K. and Rinnooy Kan, A.H.G. (1979). Optimization and approximation in deterministic sequencing and scheduling: a survey, Annals of Discrete Mathematics, 5:287-326.

Hoogeveen, J.A. (2005). Multicriteria Scheduling, European Journal of Operational Research, 167(3):592-623.

Nagar, A., Haddock, J. and Heragu, S. (1995). Multiple and bicriteria scheduling: A literature survey. European Journal of Operational Research, 81(1):88-104.

Oyetunji, E.O. (2006). The Development of Scheduling Heuristics for the Bicriteria problems on a Single Machine, PhD thesis, University of Ibadan, Nigeria.

Oyetunji, E.O. (2009). Mixed Multi Objectives Scheduling, A paper presented at the $3^{\text {rd }}$ Workshop on Mathematical and Computational Methods in Biology and Medicine (Holistic Modeling and Trends in the Biogeosciences) held at the University of Cape Coast, Cape Coast, Ghana; $21^{\text {st }}-24^{\text {th }}$ May, 2009.

Oyetunji, E.O. and Oluleye, A.E. (2007). Heuristics for minimizing total completion time on single machine with release time. Advanced Materials Research, 18-19:347352.

Oyetunji, E.O. and Oluleye, A.E. (2008a). Heuristics for Minimizing Total Completion Time and Number of Tardy Jobs Simultaneously on Single Machine with Release Time. Research Journal of Applied Sciences, 3(2):147-152.

Oyetunji, E.O. and Oluleye, A.E. (2008b). Heuristics for minimizing number of tardy jobs on Single machine with release time, South African Journal of Industrial Engineering, 19(2):183 - 196.

Stein, C. and Wein, J. (1997). On the Existence of Schedules that are Near-Optimal for both Makespan and Total Weighted Completion Time. Operations Research Letters, 21(3):115-122 